\title{
Design and Implementation of Mppt Controller with Monitoring and Control of Pv Modules Using Embedded Wireless Communication
}

\author{
R.Sakthivel, Dr.S.Ramamoorthy \\ P.G Student (Dept.Of.EEE), Dean(Dept.Of.EEE) \\ Bharath Institute Of Science And Technology
}

\begin{abstract}
To extract maximum power from the solar panel by using MPPT controller with $P \& O$ algorithm Using the effective Embedded Wireless communication technology to overcome the existing problem in manual monitoring of solar PV modules. To track and monitor the voltage, current, maximum power, solar light irradiance of PV modules and to ensure the reliability and effectiveness of the system. Use of solar panels is gaining an ever increasing foothold in society, especially on the roof of the houses for feeding domestic electrical appliances in recent times. In many situations, the expensive solar panels stop working due to some external nuisances which goes unnoticed to the users. There is a need of a low-cost monitoring system to get information of the defected solar panels for timely repair and maintenance. The design, development, and trial work of a performance monitoring system of distributed solar panels along with automated data logging based on a low-cost wireless sensors network has been reported to help the current situation. The developed system can be used up to $146 \mathrm{~V}$ and $15.5 \mathrm{~A}$ solar cell systems with automatic selection of best resolutions. The system can be extended for wide range of solar cells for material research and development activities. The fabricated system has been used for field trials and very satisfactory.
\end{abstract}

\section{Introduction}

This Public Service Commission (PSC or Commission) publication contains information that will beuseful to people who have an interest in the role of renewable energy resources in Wisconsin's electrical generation mix.

\section{Renewable Energy}

Renewable energy refers to energy from a source that is continuously replenished by natural processes. State law (Wis. Stat. §196.378(1)) Supply Utilities and independent power producers are researching ways to expand the use of renewable resources. One of the most important benefits of renewable resources is their longterm availability.

Another important benefit is their minimal impact to the atmosphere. These technologies have not been associated with mercury emissions or the causes of acid rain and have little or no negative impact on climate change. As part of 2005 Wisconsin Act 141, the Wisconsin Legislature established the current renewable portfolio standard (RPS), requiring each investor-owned electric utility, municipal electric utility and rural electric cooperative (electric providers) to meet a gradually increasing percentage of their retail sales with qualified renewable resources. The current RPS establishes the goal that by the end of 2015,10 percent of all electric energy consumed in the state will be renewable energy.

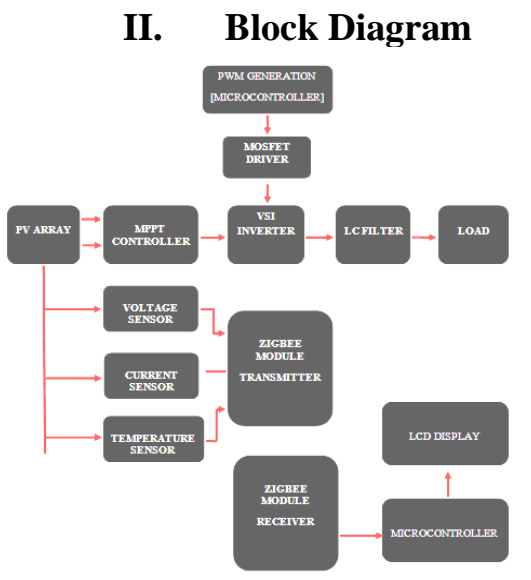




\section{THEORY AND CONSTRUCTION}

Solar panels use light energy (photons) from the sun to generate electricity through the photovoltaic effect. The structural (load carrying) member of a module can either be the top layer or the back layer. The majority of modules use wafer-based crystalline silicon cells or thin-film cells based on cadmium telluride or silicon. The conducting wires that take the current off the panels may contain silver, copper or other conductive (but generally not magnetic) transition metals. The cells must be connected electrically to one another and to the rest of the system. Cells must also be protected from mechanical damage and moisture. Most solar panels are rigid, but semi-flexible ones are available, based on thin-film cells. Electrical connections are made in series to achieve a desired output voltage and/or in parallel to provide a desired current capability. Separate diodes may be needed to avoid reverse currents, in case of partial or total shading, and at night. The p-n junctions of monocrystalline silicon cells may have adequate reverse current characteristics that these are not necessary. Reverse currents waste power and can also lead to overheating of shaded cells. Solar cells become less efficient at higher temperatures and installers try to provide good ventilation behind solar panels.

Some recent solar panel designs include concentrators in which light is focused by lenses or mirrors onto an array of smaller cells. This enables the use of cells with a high cost per unit area (such as gallium arsenide) in a cost-effective way

Depending on construction, photovoltaic panels can produce electricity from a range of frequencies of light, but usually cannot cover the entire solar range (specifically, ultraviolet, infrared and low or diffused light). Hence much of the incident sunlight energy is wasted by solar panels, and they can give far higher efficiencies if illuminated with monochromatic light.

Therefore another design concept is to split the light into different wavelength ranges and direct the beams onto different cells tuned to those ranges. This has been projected to be capable of raising efficiency by $50 \%$. The use of infrared photovoltaic cells has also been proposed to increase efficiencies, and perhaps produce power at night. Currently the best achieved sunlight conversion rate (solar panel efficiency) is around $21 \%$ in commercial product, typically lower than the efficiencies of their cells in isolation. The Energy Density of a solar panel is the efficiency described in terms of peak power output per unit of surface area, commonly expressed in units of Watts per square foot $(\mathrm{W} / \mathrm{ft} 2)$.

\section{Maximum Power Point Tracker:}

A maximum power point tracker (or MPPT) is a high efficiency DC to DC converter that presents an optimal electrical load to a solar panel or array and produces a voltage suitable for the load. PV cells have a single operating point where the values of the current (I) and Voltage (V) of the cell result in a maximum power output. These values correspond to a particular load resistance, which is equal to V/I as specified by Ohm's Law. The power $\mathrm{P}$ is given by $P=V^{*} I$. A PV cell has an exponential relationship between current and voltage, and the maximum power point (MPP) occurs at the knee of the curve where $\mathrm{d} P / \mathrm{d} V=0$. At this point the characteristic resistance equals that of the load resistance. Maximum power point trackers utilize some type of control circuit or logic to search for this point and thus to allow the converter circuit to extract the maximum power available from a cell. Traditional solar inverters perform MPPT for an entire array as a whole. In such systems the same current, dictated by the inverter, flows though all panels in the string. But because different panels have different IV curves, i.e. different MPPs (due to manufacturing tolerance, partial shading, etc.) this architecture means some panels will be performing below their MPP, resulting in the loss of energy.

Some companies (see power optimizer) are now placing peak power point converters into individual panels, lallowing each to operate at peak efficiency despite uneven shading, soiling or electrical mismatch. At night, an off-grid pv power system uses batteries to supply its loads. Although the battery pack voltage when fully charged may be close to the pv array's peak power point, this is unlikely to be true at sunrise when the battery is partially discharged. Charging may begin at a voltage considerably below the array peak power point, and a mppt can resolve this mismatch. When the batteries in an off-grid system are full and pv production exceeds local loads, a mppt can no longer operate the array at its peak power point as the excess power has nowhere to go. The mppt must then shift the array operating point away from the peak power point until production exactly matches demand. (an alternative approach commonly used in spacecraft is to divert surplus pv power into a resistive load, allowing the array to operate continuously at its peak power point.)

In a grid-tied photovoltaic system, the grid is essentially a battery with near infinite capacity. The grid can always absorb surplus PV power, and it can cover shortfalls in PV production (e.g., at night). Batteries are thus needed only for protection from grid outages. The MPPT in a grid tied PV system will always operate the array at its peak power point unless the grid fails when the batteries are full and there are insufficient local loads. It would then have to back the array away from its peak power point as in the off-grid case (which it has temporarily become).

MPPTs can be designed to drive an electric motor without a storage battery. They provide significant advantages, especially when starting a motor under load. This can require a starting current that is well above 
the short-circuit rating of the PV panel. A MPPT can step the panel's relatively high voltage and low current down to the low voltage and high current needed to start the motor. Once the motor is running and its current requirements have dropped, the MPPT will automatically increase the voltage to normal. In this application, the MPPT can be seen as an electrical analogue to the transmission in a car; the low gears provide extra torque to the wheels until the car is up to speed.

\subsection{MAXIMUM POWER POINT TRACKING ALGORITHMS \\ AN OVERVIEW OF MAXIMUM POWER POINT TRACKING}

A typical solar panel converts only 30 to 40 percent of the incident solar irradiation into Electrical energy. Maximum power point tracking technique is used to improve the efficiency of the solar panel. According to Maximum Power Transfer theorem, the power output of a circuit is maximum, when the Thevenin impedance of the circuit (source impedance) matches with the load impedance. Hence our problem of tracking the maximum power point reduces to an impedance matching problem. In the source side we are using a boost convertor connected to a solar panel in order to enhance the output voltage so that it can be used for different applications like motor load. By changing the duty cycle of the boost converter appropriately we can match the source impedance with that of the load impedance.

\subsection{DIFFERENT MPPT TECHNIQUES}

There are different techniques used to track the maximum power point. Few of the most popular techniques are:

1) Perturb and observe (hill climbing method)

2) Incremental Conductance method

3) Fractional short circuit current

4) Fractional open circuit voltage

5) Neural networks

6) Fuzzy logic

The choice of the algorithm depends on the time complexity the algorithm takes to track the MPP, implementation cost and the ease of implementation

\subsection{PERTURB \& OBSERVE}

Perturb \& Observe $(\mathrm{P} \& \mathrm{O})$ is the simplest method. In this we use only one sensor, that is the voltage sensor, to sense the PV array voltage and so the cost of implementation is less and hence easy to implement. The time complexity of this algorithm is very less but on reaching very close to the MPP it doesn't stop at the MPP and keeps on perturbing on both the directions. When this happens the algorithm has reached very close to the MPP and we can set an appropriate error limit or can use a wait function which ends up increasing the time complexity of the algorithm. However the method does not take account of the rapid change of irradiation level (due to which MPPT changes) and considers it as a change in MPP due to perturbation and ends up calculating the wrong MPP. To avoid this problem we can use incremental conductance method.

\subsection{INCREMENTAL CONDUCTANCE}

Incremental conductance method uses two voltage and current sensors to sense the output voltage and current of the PV array.

At MPP the slope of the PV curve is 0.

$(\mathrm{dP} / \mathrm{dV}) \mathrm{MPP}=\mathrm{d}(\mathrm{VI}) / \mathrm{dV}(4.1)$

$0=\mathrm{I}+\mathrm{VdI} / \mathrm{dVMPP}(4.2)$

$\mathrm{DI} / \mathrm{dVMPP}=-\mathrm{I} / \mathrm{V}$

The left hand side is the instantaneous conductance of the solar panel. When this instantaneous conductance equals the conductance of the solar then MPP is reached. Here we are sensing both the voltage and current simultaneously. Hence the error due to change in irradiance is eliminated. However the complexity and the cost of implementation increase. As we go down the list of algorithms the complexity and the cost of implementation goes on increasing which may be suitable for a highly complicated system. This is the reason that Perturb and Observe and Incremental Conductance method are the most widely used algorithms. Owing to its simplicity of implementation we have chosen the Perturb \& Observe algorithm for our study among the two.

\subsection{FRACTIONAL OPEN CIRCUIT VOLTAGE}

The near linear relationship between VMPP and VOC of the PV array, under varying irradiance and temperature levels, has given rise to the fractional VOC method. VMPP = k1 Voc (4.4) where $\mathrm{k} 1$ is a constant of proportionality. Since k1 is dependent on the characteristics of the PV array being used, it usually has to be computed beforehand by empirically determining VMPP and VOC for the specific PV array at different 
irradiance and temperature levels. The factor $\mathrm{k} 1$ has been reported to be between 0.71 and 0.78 . Once $\mathrm{k} 1$ is known, VMPP can be computed with VOC measured periodically by momentarily shutting down the power converter. However, this incurs some disadvantages, including temporary loss of power.

\subsection{FRACTIONAL SHORT CIRCUIT CURRENT}

Fractional ISC results from the fact that, under varying atmospheric conditions, IMPP is

Approximately linearly related to the ISC of the PV array. IMPP $=\mathbf{K} 2 \mathbf{I s c}$ (4.5) where K2 is a proportionality constant. Just like in the fractional VOC technique, $\mathrm{K} 2$ has to be determined according to the PV array in use. The constant K2 is generally found to be between 0.78 and 0.92 . Measuring ISC during operation is problematic. An additional switch usually has to be added to the power converter to periodically short the PV array so that ISC can be measured using a current sensor

\subsection{FUZZY LOGIC CONTROL}

Microcontrollers have made using fuzzy logic control popular for MPPT over last decade. Fuzzy logic controllers have the advantages of working with imprecise inputs, not needing an accurate mathematical model, and handling nonlinearity

\subsection{NEURAL NETWORK}

Another technique of implementing MPPT which are also well adapted for microcontrollers is neural networks. Neural networks commonly have three layers: input, hidden, and output layers. The number nodes in each layer vary and are user-dependent. The input variables can be PV array parameters like VOC and ISC, atmospheric data like irradiance and temperature, or any combination of these. The output is usually one or several reference signals like a duty cycle signal used to drive the power converter to operate at or close to the MPP

\subsection{PERTURB \& OBSERVE ALGORITHM}

The Perturb \& Observe algorithm states that when the operating voltage of the PV panel is perturbed by a small increment, if the resulting change in power_P is positive, then we are going in the direction of MPP and we keep on perturbing in the same direction. If_P is negative, we are going away from the direction of MPP and the sign of perturbation supplied has to be changed

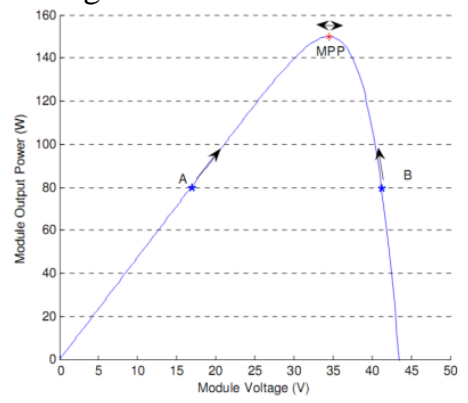

\section{Voltage Source Inverters:}

The main objective of static power converters is to produce an ac output waveform from a dc power supply. These are the types of waveforms required in adjustable speed drives (ASDs), uninterruptible power supplies (UPS), static var compensators, active filters, flexible ac transmission systems (FACTS), and voltage compensators, which are only a few applications. For sinusoidal ac outputs, the magnitude, frequency, and phase should be controllable.

According to the type of ac output waveform, these topologies can be considered as voltage source inverters (VSIs), where the independently controlled ac output is a voltage waveform. These structures are the most widely used because they naturally behave as voltage sources as required by many industrial applications, such as adjustable speed drives (ASDs), which are the most popular application of inverters. Similarly, these topologies can be found as current source inverters (CSIs), where the independently controlled ac output is a current waveform. These structures are still widely used in medium-voltage industrial applications, where highquality voltage waveforms are required. Static power converters, specifically inverters, are constructed from power switches and the ac output waveforms are therefore made up of discrete values. This leads to the generation of waveforms that feature fast transitions rather than smooth ones.

For instance, the ac output voltage produced by the VSI of a standard ASD is a three-level waveform .Although this waveform is not sinusoidal as expected its fundamental component behaves as such. This behavior should be ensured by a modulating technique that controls the amount of time and the sequence used to 
switch the power valves on and off. The modulating techniques most used are the carrier-based technique (e.g., sinusoidal pulse width modulation, SPWM), the space-vector (SV) technique, and the selective-harmonicelimination (SHE) technique.
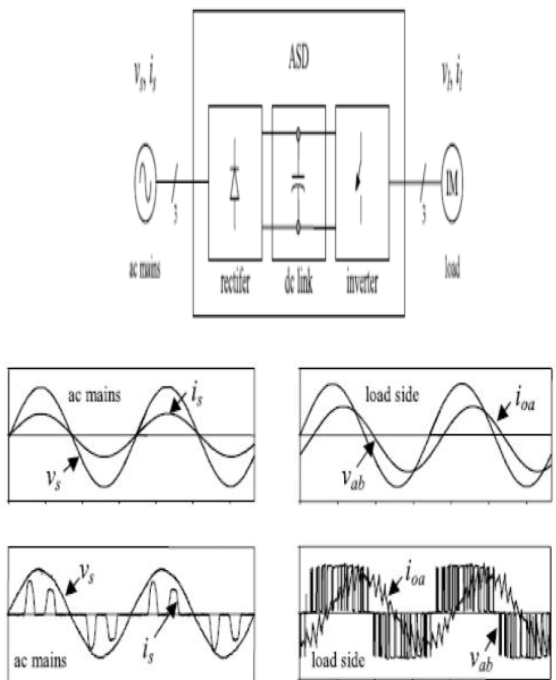

The ac output voltage produced by the VSI of a standard ASD

a) The electrical power conversion topology;

b) The ideal input (ac mains) and output (load) waveforms; and

c) The actual input (ac mains) and output (load) waveforms.

\subsection{SINGLE-PHASE VOLTAGE SOURCE INVERTERS:}

Single-phase voltage source inverters (VSIs) can be found as half-bridge and full-bridge topologies. Although the power range they cover is the low one, they are widely used in power supplies, single-phase UPSs, and currently to form elaborate high-power static power topologies, such as for instance, the multi cell configurations.

\subsection{HALF-BRIDGE VSI:}

The power topology of a half-bridge VSI, consists of two large capacitors are required to provide a neutral point $\mathrm{N}$, such that each capacitor maintains a constant voltage (Vi)/2. Because the current harmonics injected by the operation of the inverter are low-order harmonics, a set of large capacitors (C+ and C-) is required. It is clear that both switches S+ and S- cannot be ON simultaneously because a short circuit across the dc link voltage source Vi would be produced. There are two defined (states 1 and 2) and one undefined (state 3) switch state as In order to avoid the short circuit across the dc bus and the undefined ac output voltage condition, the modulating technique should always ensure that at any instant either the top or the bottom switch of the inverter leg is on.

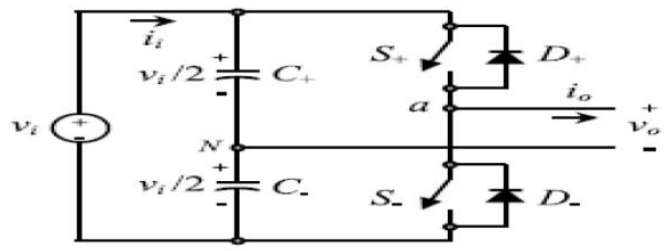

\subsection{FULL-BRIDGE VSI:}

This inverter is similar to the half-bridge inverter; however, a second leg provides the neutral point to the load. As expected, both switches S1+ and S1- (or S2+ and S2-) cannot be on simultaneously because a short circuit across the dc link voltage source Vi would be produced. There are four defined (states 1, 2, 3, and 4) and one undefined (state 5) switch states. The undefined condition should be avoided so as to be always capable of defining the ac output voltage. It can be observed that the ac output voltage can take values up to the dc link value $\mathrm{Vi}$, which is twice that obtained with half-bridge VSI topologies. Several modulating techniques have been developed that are applicable to full-bridge VSIs. Among them are the PWM (bipolar and unipolar) techniques. 


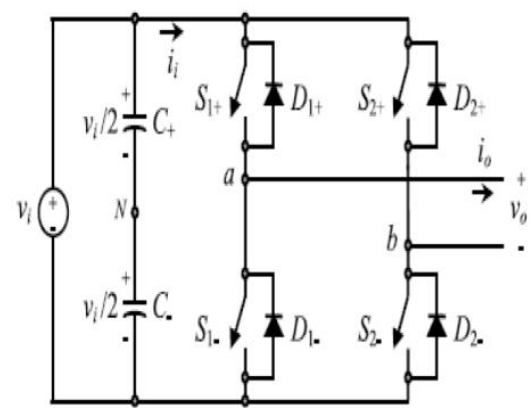

\subsection{THREE PHASE VOLTAGE SOURCE INVERTERS:}

Single-phase VSIs cover low-range power applications and three-phase VSIs cover the medium- to high-power applications. The main purpose of these topologies is to provide a three-phase voltage source, where the amplitude, phase, and frequency of the voltages should always be controllable. Although most of the applications require sinusoidal voltage waveforms (e.g., ASDs, UPSs, FACTS, VAR compensators), arbitrary voltages are also required in some emerging applications (e.g., active filters, voltage compensators).

The standard three-phase VSI topology is shown and the eight valid switch states. As in single-phase VSIs, the switches of any leg of the inverter (S1 and S4, S3 and S6, or S5 and S2) cannot be switched on simultaneously because this would result in a short circuit across the dc link voltage supply. Similarly, in order to avoid undefined states in the VSI, and thus undefined ac output line voltages, the switches of any leg of the inverter cannot be switched off simultaneously as this will result in voltages that will depend upon the respective line current polarity. Of the eight valid states, two of them produce zero ac line voltages. In this case, the ac line currents freewheel through either the upper or lower components. The remaining states produce non-zero ac output voltages. In order to generate a given voltage waveform, the inverter moves from one state to another. Thus the resulting ac output line voltages consist of discrete values of voltages that are $\mathrm{Vi}, 0$, and $-\mathrm{Vi}$ for the topology. The selection of the states in order to generate the given waveform is done by the modulating technique that should ensure the use of only the valid states.

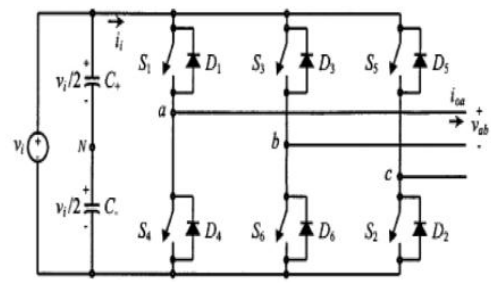

\section{EVOLUTION OF LR-WPAN STANDARDIZATION \\ VI. Zigbee:}

The cellular network was a natural extension of the wired telephony network that became pervasive during the mid-20th century. As the need for mobility and the cost of laying new wires increased,the motivation for a per sonal connection independent of location to that network also increased.Coverage of large area is provided through $(1-2 \mathrm{~km})$ cells that cooperate with their neighbors to create a seemingly seamless network. Examples of standards are GSM, IS-136, IS-95. Cellular standards basically aimed at facilitating voice communications throughout a metropolitan area.During the mid-1980s, it turned out that an even smaller coverage area is needed for higher user densities and the emergent data traffic. The IEEE 802.11 working group for WLANs is formed to create a wireless local area network standard. Whereas IEEE 802.11 was concerned with features such as Ethernet matching speed, longrange $(100 \mathrm{~m})$, complexity to handle seamless roaming, message forwarding, and data throughput of 2-11Mbps, WPANs are focused on a space around a person or object that typically extends up to $10 \mathrm{~m}$ in all directions. The focus of WPANs is low-cost, low power, short range and very small size. The IEEE 802.15 working group is formed to create WPAN standard. This group has currently defined three classes of WPANs that are differentiated by data rate, battery drain and quality of service(QoS). The high data rate WPAN(IEEE 802.15.3) is suitable for multi-media applications that require very high QoS. Medium rate WPANs (IEEE 802.15.1/Blueetooth) will handle a variety of tasks ranging from cell phones to PDA communications and have QoS suitable for voice communications. The low rate WPANs(IEEE 802.15.4/LR-WPAN) is intended to serve a set of industrial, residential and medical applications with very low power consumption and cost requirement not considered by the aboveWPANs and with relaxed needs for data rate and QoS. The low data rate enables the LR-WPAN to consume very little power. 


\subsection{ZIGBEE AND IEEE 802.15.4}

ZigBee technology is a low data rate, low power consumption, low cost, wireless networking protocol targeted towards automation and remote control applications. IEEE 802.15.4 committee started working on a low data rate standard a short while later. Then the ZigBee Alliance and the IEEE decided to join forces and ZigBee is the commercial name for this technology.ZigBee is expected to provide low cost and low power connectivity for equipment that needs battery life as long as several months to several years but does not require data transfer rates as high as those enabled by Bluetooth. In addition, ZigBee can be implemented in mesh networks larger than is possible with Bluetooth. ZigBee compliant wireless devices are expected to transmit 1075 meters, depending on the RF environment and the power output consumption required for a given application, and will operate in the unlicensed RF worldwide $(2.4 \mathrm{GHz}$ global, $915 \mathrm{MHz}$ Americas or $868 \mathrm{MHz}$ Europe). The data rate is $250 \mathrm{kbps}$ at $2.4 \mathrm{GHz}, 40 \mathrm{kbps}$ at $915 \mathrm{MHz}$ and $20 \mathrm{kbps}$ at $868 \mathrm{MHz}$. IEEE and ZigBee Alliance have been working closely to specify the entire protocol stack. IEEE 802.15 .4 focuses on the specification of the lower two layers of the protocol(physical and data link layer). On the other hand, ZigBee Alliance aims to provide the upper layers of the protocol stack (from network to the application layer) for interoperable data networking, security services and a range of wireless home and building control solutions, provide interoperability compliance testing, marketing of the standard, advanced engineering for the evolution of the standard. This will assure consumers to buy products from different manufacturers with confidence that the products will work together.

IEEE 802.15.4 is now detailing the specification of PHY and MAC by offering building blocks for different types of networking known as "star, mesh, and cluster tree". Network routing schemes are designed to ensure power conservation, and low latency through guaranteed time slots. A unique feature of ZigBee network layer is communication redundancy eliminating "single point of failure" in mesh networks. Key features of PHY include energy and link quality detection, clear channel assessment for improved coexistence with other wireless networks.

\subsection{ZIGBEE VS. BLUETOOTH}

ZigBee looks rather like Bluetooth but is simpler, has a lower data rate and spends most of its time snoozing. This characteristic means that a node on a ZigBee network should be able to run for six months to two years on just two AA batteries. (HOW?) The operational range of ZigBee is $10-75 \mathrm{~m}$ compared to $10 \mathrm{~m}$ for Bluetooth(without a power amplifier).ZigBee sits below Bluetooth in terms of data rate. The data rate of ZigBee is $250 \mathrm{kbps}$ at $2.4 \mathrm{GHz}, 40 \mathrm{kbps}$ at $915 \mathrm{MHz}$ and $20 \mathrm{kbps}$ at $868 \mathrm{MHz}$ whereas that of Bluetooth is $1 \mathrm{Mbps}$.ZigBee uses a basic master-slave configuration suited to static star networks of many infrequently used devices that talk via small data packets. It allows up to 254 nodes. Bluetooth's protocol is more complex since it is geared towards handling voice, images and file transfers in ad hoc networks. Bluetooth devices can support scatternets of multiple smaller non-synchronized networks(piconets). It only allows up to 8 slave nodes in a basic masterslave piconet set-up. When ZigBee node is powered down, it can wake up and get a packet in around $15 \mathrm{msec}$ whereas a Bluetooth device would take around $3 \mathrm{sec}$ to wake up and respond.

\section{IEEE 802.15.4WPAN}

The main features of this standard are network flexibility, low cost, very low power consumption, and low data rate in an adhoc self-organizing network among inexpensive fixed, portable and moving devices. It is developed for applications with relaxed throughput requirements which cannot handle the power consumption of heavy protocol stacks.

\section{Components of WPAN}

A ZigBee system consists of several components. The most basic is the device. A device can be a full-function device (FFD) or reduced-function device (RFD). A network shall include at least one FFD, operating as the PAN coordinator.The FFD can operate in three modes: a personal area network (PAN) coordinator, a coordinator or a device. An RFD is intended for applications that are extremely simple and do not need to send large amounts of data. An FFD can talk to RFDs or FFDs while an RFD can only talk to an FFD.

\subsection{NETWORK TOPOLOGIES}

Figure 2.1 shows 3 types of topologies that ZigBee supports: star topology, peer-to-peer topology and cluster tree.

\subsection{STAR TOPOLOGY}

In the star topology, the communication is established between devices and a single central controller, called the PAN coordinator. The PAN coordinator may be mains powered while the devices will most likely be battery powered. Applications that benefit from this topology include home automation, personal computer (PC) 
peripherals, toys and games. After an FFD is activated for the first time, it may establish its own network and become the PAN coordinator. Each start network chooses a PAN identifier, which is not currently used by any other network within the radio sphere of influence. This allows each star network to operate independently.

\subsection{PEER-TO-PEER TOPOLOGY}

In peer-to-peer topology, there is also one PAN coordinator. In contrast to star topology, any device can communicate with any other device as long as they are in range of one another. A peer-to-peer
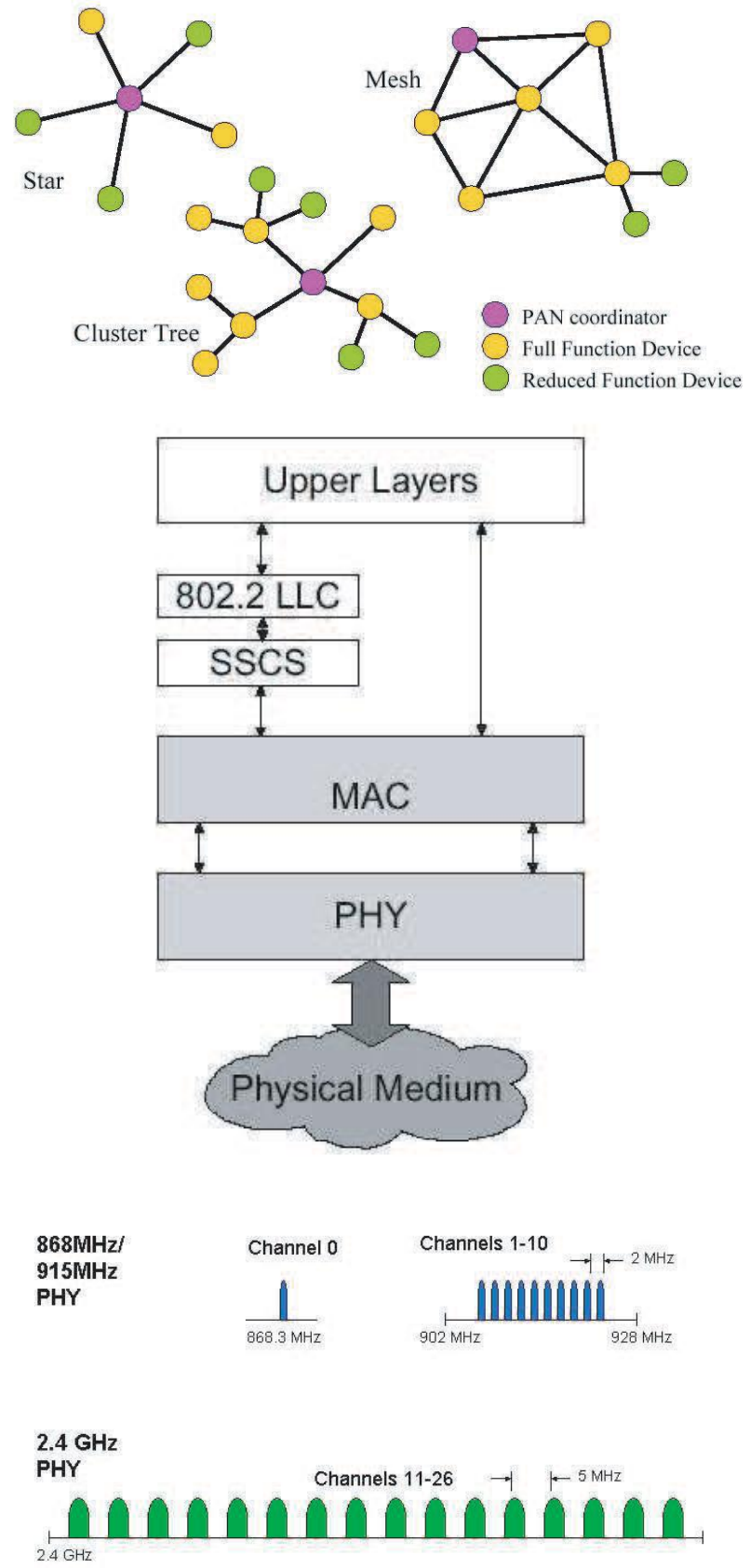

The PHY provides two services: the PHY data service and PHY management service interfacing to the physical layer management entity (PLME). The PHY data service enables the transmission andreception of PHY protocol data units (PPDU) across the physical radio channel. The features of the PHY are activation and deactivation of the radio transceiver, energy detection (ED), link quality indication (LQI), channel selection, clear channel assessment (CCA) and transmitting as well as receiving packets across the physical medium.The standard offers two PHY options based on the frequency band. Both are based on direct sequence spread spectrum (DSSS). The data rate is $250 \mathrm{kbps}$ at $2.4 \mathrm{GHz}, 40 \mathrm{kbps}$ at $915 \mathrm{MHz}$ and $20 \mathrm{kbps}$ at $868 \mathrm{MHz}$. The higher data rate at $2.4 \mathrm{GHz}$ is attributed to a higher-order modulation scheme. Lower frequency provide longer range due to lower propagation losses. Low rate can be translated into better sensitivity and larger coverage area. Higher rate means higher throughput, lower latency or lower duty cycle. 
There is a single channel between 868 and $868.6 \mathrm{MHz}, 10$ channels between 902.0 and $928.0 \mathrm{MHz}$, and 16 channels between 2.4 and $2.4835 \mathrm{GHz}$ as shown in Figure 3.2. Several channels in different frequency bands enables the ability to relocate within spectrum. The standard also allows dynamic channel selection, a scan function that steps through a list of supported channels in search of beacon, receiver energy detection, link quality indication, channel switching. Receiver sensitivities are $-85 \mathrm{dBm}$ for $2.4 \mathrm{GHz}$ and $-92 \mathrm{dBm}$ for $868 / 915 \mathrm{MHz}$. The advantage of $6-8 \mathrm{~dB}$ comes from the advantage of lower rate. The achievable range is a function of receiver sensitivity and transmit power. The maximum transmit power shall conform with local regulations. A compliant device shall have its nominal transmit power level indicated by the PHY parameter, phyTransmitPower

\section{Circuit Diagram:}
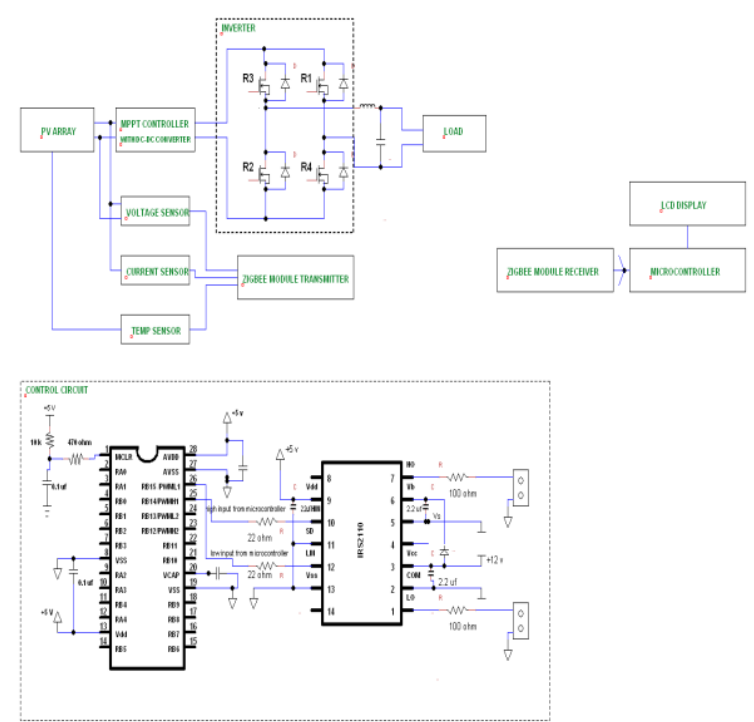

A microcontroller- based standalone PV cell monitoring system has been reported in the above circuit, which can supply power to the wireless sensor node. Making the control and monitoring system capable of communicating through a wireless medium adds the flexibility. A system has been developed which can track and monitor the performance of the solar panel, can set the maximum power point of solar panels, monitor its instantaneous power output, and send and receive data and commands from a operator who is based on a remote location using wireless medium. When generating the idea for this project, the likely future scenario is considered where power companies or communities have placed a network of solar panels to provide electricity to households. They would like to control and monitor the solar panels network from the work base. Making the control and monitoring system capable of communicating through a wireless medium adds the flexibility that the technician does not have to be in the actual area where solar panels are located to monitor the solar panel networks.

In the current study, a system has been developed which can track and monitor the performance of the solar panel, can set the maximum power point of solar panels, monitor its instantaneous power output, and send and receive data and commands from a operator who is based on a remote location using wireless medium. The system will also be able to monitor whether there is any operational problem of the sola $r$ panel, such as not generating any output even in the presence of sunlight. The problem can be due to a cell problem itself or may be due to shading (a branch of a fallen tree might have created the shading). The system can give a warning sound by which the household people may physically observe it to know the problem. The conceptual diagram is shown in Fig. 2. All the parameters are measured by the local measurement circuit and then transferred using the Zigbee communication protocol. The reasons for using Zigbee are that it matched the data rate, distance and power consumption and cost compared to other communication protocols available.The important information are the open circuit voltage (V), short circuit current, and the maximum power point (MPP, ), and the associated voltage and current at this maximum power point (V and, respectively). The MPP and associated values can vary significantly, depending on solar irradiance intensity, cell type, temperature, shading, etc. This type of measurement is also invaluable in PV system maintenance, where it is necessary to detect faulty cells and panels (a single faulty or shaded cell can significantly destroy the output of an entire panel or array), and also useful for insuring panels in arrays are matched so as to get maximum power.Many commercial I-V curve tracers exist, some being purposely designed for PV market or the adaptation of common I-V curve tracer equipment as is shown in Table I. 
These range in price from NZ \$1620 for the Prova 200 to NZ \$32 100 for the Daystar DS-100C. Generally, these have PC link for control and data download, and have inputs for or come with irradiance and temperature sensors. Usually, most of the available commercial I-V-P curve tracer are designed for desktop applications and can monitor the performance of one solar panel at a time. The purpose of the work is to design and develop wireless sensor network which can monitor performances of a few solar panels simultaneously with the help of switching arrangement and provide valuable information about failure and/or maintenance data. The challenge was to develop each wireless remote sensing and monitoring unit under NZ $\$ 200.00$ for a $150 \mathrm{~V}, 15$ A solar panel system including communication devices. The design and fabrication of each remote system has been soarried mut mithin the nrine

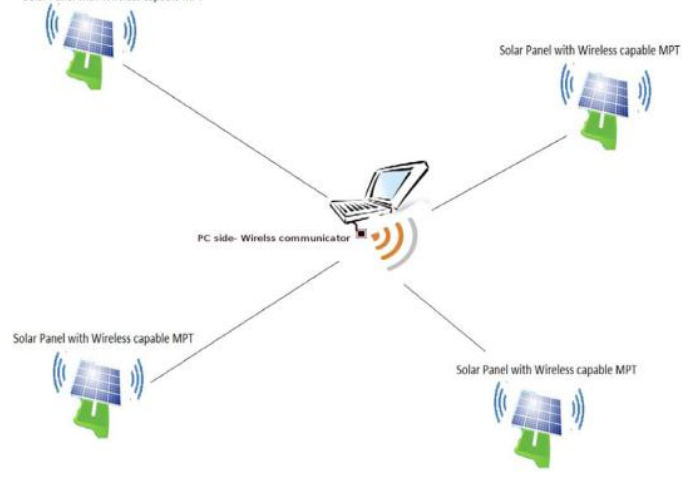

MATLAB is a high-performance language for technical computing. It integrates computation, visualization, and programming in an easy-to-use environment where problems and solutions are expressed in familiar mathematical notation. Typical uses include

- Math and computation.

- Algorithm development.

- Data acquisition.

- Modeling, simulation, and prototyping.

- Data analysis, exploration, and visualization.

- Scientific and engineering graphics.

- Application development, including graphical user interface building.

MATLAB is an interactive system whose basic data element is an array that does not require dimensioning. This allows you to solve many technical computing problems, especially those with matrix and vector formulations, in a fraction of the time it would take to write a program in a scalar non interactive language such as $\mathrm{C}$ or FORTRAN.

The name MATLAB stands for matrix laboratory. MATLAB was originally written to provide easy access to matrix software developed by the LINPACK and EISPACK projects. Today, MATLAB engines incorporate the LAPACK and BLAS libraries, embedding the state of the art in software for matrix computation MATLAB has evolved over a period of years with input from many users. In university environments, it is the standard instructional tool for introductory and advanced courses in mathematics, engineering, and science. In industry, MATLAB is the tool of choice for high-productivity research, development, and analysis.

MATLAB features a family of add-on application-specific solutions called toolboxes .Very important to most users of MATLAB, toolboxes allow you to learn and apply specialized technology. Toolboxes are comprehensive collections of MATLAB functions (M-files) that extend the MATLAB environment to solve particular classes of problems. Areas in which toolboxes are available include signal processing, control Systems, neural networks, fuzzy logic, wavelets, simulation, and many others.

\section{Simpower Systems:}

Sim Power Systems and other products of the Physical Modeling product family work together with Simulink to model electrical, mechanical, and control systems. Sim Power Systems operates in the Simulink environment. Therefore, before starting this user's guide, you should be familiar with Simulink.

\subsection{THE ROLE OF SIMULATION IN DESIGN:}

Electrical power systems are combinations of electrical circuits and electromechanical devices like motors and generators. Engineers working in this discipline are constantly improving the performance of the systems. Requirements for drastically increased efficiency have forced power system designers to use power electronic devices and sophisticated control system concepts that tax traditional analysis tools and techniques. Further complicating the analyst's role is the fact that the system is often so nonlinear that the only way to 
understand it is through simulation .Land-based power generation from hydroelectric, steam, or other devices is not the only use of power systems. A common attribute of these systems is their use of power electronics and control systems to achieve their performance

Sim Power Systems is a modern design tool that allows scientists and engineers to rapidly and easily build models that simulate power systems. Sim Power Systems uses the Simulink environment, allowing you to build a model using simple click and drag procedures. Not only can you draw the circuit topology rapidly, but your analysis of the circuit can include its interactions with mechanical, thermal, control, and other disciplines. This is possible because all the electrical parts of the simulation interact with the extensive Simulink modeling library. Since Simulink uses MATLAB ${ }^{\circledR}$ as its computational engine, designers can also use MATLAB toolboxes and Simulink block sets. Sim Power Systems and Sim Mechanics share a special Physical Modeling block and connection line interface.

\subsection{SIMPOWER SYSTEMS LIBRARIES}

The libraries contain models of typical power equipment such as transformers, lines, machines, and power electronics. The capabilities of Sim Power Systems for modeling a typical electrical system are illustrated in demonstration files. And for users who want to refresh their knowledge of power system theory, there are also self-learning case studies. The Sim Power Systems main library, power lib, organizes its blocks into libraries according to their behavior. The power library window displays the block library icons and names. Double-click a library icon to open the library and access the blocks. The main Sim Power Systems power library window also contains the Powergui block that opens a graphical user interface for the steady-state analysis of electrical circuits. This is possible because all the electrical parts of the simulation interact with the extensive Simulink modeling library. Since Simulink uses MATLAB as its computational engine, designers can also use MATLAB toolboxes and Simulink block sets. Sim Power Systems and Sim Mechanics share a special Physical Modeling block and connection line interface.

\subsection{SIMULATION DIAGRAM:}

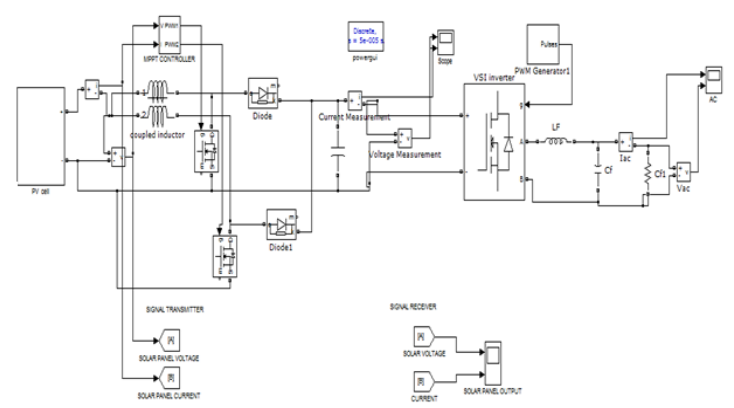

\subsection{SIMULATION RESULT:} SOLAR PANEL OUTPUT:

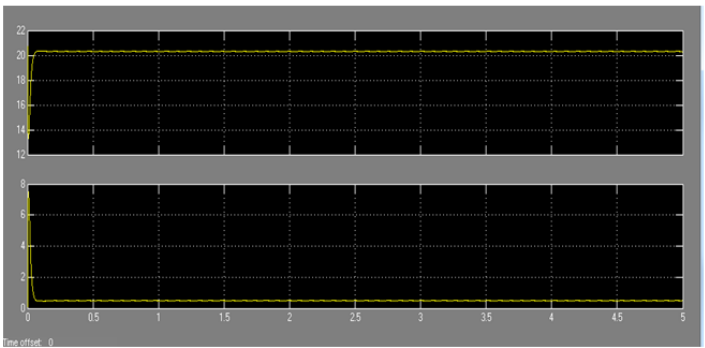




\subsection{OUTPUT:}

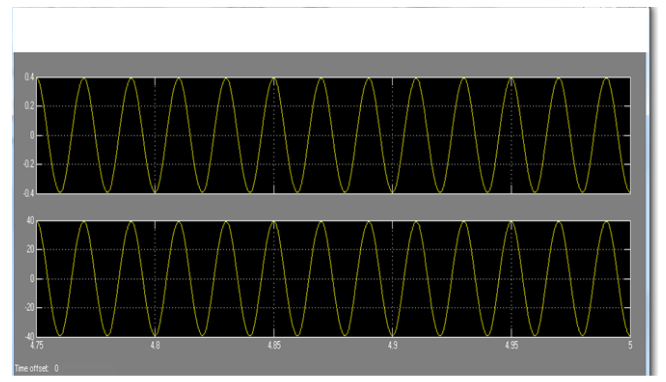

\section{Conclusion:}

A wireless sensor network-based performance monitoring system has been developed for distributed solar panels. The developed system offers the following advantages.

- Can also be used as a standalone current and a voltage measuring transducer.

- Integration of pyranometer also allowed both efficiency calculations and monitoring of atmospheric

light changes during scan.

- Adjustable scan speeds eliminate $50 \mathrm{~Hz} \mathrm{AC}$ interference from solar simulator.

- Use of filters to allow DC values from AC signals from MPP controllers.

- Small portable standalone microprocessor-based system.

- Isolated (i.e., isolation amps or digital isolation etc.).

The developed system will provide a low-cost solution for the regular maintenance information of the solar panel installed on the roof of the houses. The developed system is flexible and can be used for any other performance monitoring system using wireless sensors and Zigbee communication. The developed system has been demonstrated at the 2010 International Digital Signal Processing Creative Design Contest held in Tainan, Taiwan, on 19 November 2010, and a prize was awarded.

\section{References}

[1] G. TamizhMani, "Testing the reliability and safety of photovoltaic modules: Failure rates and temperature effects," 2010. [Online]. Available: http://www.tuv.com/media/usa/aboutus_1/pressreleases/PTL_Magazine_Article.pdf

[2] P. Singer, "The quest for grid parity," Photovoltaics World, p. 6, 2009.

[3] M. A. Green, K. Emery, Y. Hishikawa, and W. Warta, "Solar cell efficiency tables (version 34)," Progress in Photovoltaics, vol. 17 , pp. 320-326, 2009.

[4] "PC printer port controls I-V curve tracer (an253).” 2004. [Online]. Available: www.maxim-ic/an253

[5] R. N. Briskman and P. E. Livingstone, "A low cost, user-friendly photovoltaic cell curve tracer," Solar Energy Materials and Solar Cells, vol. 46, pp. 187-199, 1997.

[6] O. Go, H. Katsuya, and N. Shigeyasu, "Development of a high-speed system measuring a maximum power point of PV modules," in Proc. IEEE 4th World Conf. Record.Photovoltaic Energy Conversion, 2006, pp. 2262-2263.

[7] T. H. Warner and C. H. Cox, III, "A high power current-voltage curve tracer employing a capacitive load," Solar Cells, vol. 7, pp. $175-181,1982$.

[8] F. Adamo, F. Attivissimo, and M. Spadavecchio, "A tool for photovoltaic panels modeling and testing," in Proc. 2010 IEEE Int. Instrum. Meas. Technol. Conf. (I2MTC 2010), Austin, TX, May 3-5, 2010, pp. 1463-1466.

[9] B. Lo, R. Phelps, and S. Michael, "Evaluation and testing of the solar cell measurement system onboard the naval postgraduate school satellite NPSAT1," in Proc. 22nd AIAA Int. Commun. Satellite Syst. Conf. Exhibit (ICSSC), Monterey, CA, May 9-12, 2004, pp. $1-8$.

[10] W. Luo, "A solar panels automatic tracking system based on OMRON PLC," in Proc. 7th Asian Control Conf., Hong Kong, China, Aug. 27-29, 2009, pp. 1611-1614.

[11] C. R. Griesbach, "Fault-tolerance solar array control using digital signal processing for peak power tracking," in Proc. 31st Intersociety Energy Conversion Eng. Conf., 1996, vol. 1, pp. 260-265, DOI: 10.1109/IECEC.1996.552881.

[12] A. J. Arustizabad, C. A. Arredondo, J. Hernandez, and G. Gordillo,"Development of equipment for monitoring PV power plants, using virtual instrumentation," in Proc. 4th IEEE World Conf. Photovoltaic Energy Conversion, 2006, vol. 2, pp. 2367-2370, DOI: 10.1109/WCPEC. 2006.279667.

[13] J. Zhu, Y. Qiu, T. R. Betts, and R. Gottschalg, "Outlier identification in outdoor measurement data-Effects of different strategies on the performance descriptors of photovoltaic modules," in Proc. 34th IEEE Photovoltaic Specialists Conf. (PVSXC), 2009, pp. $828-833$.

[14] A. H. Arianfar, M. H. M. Jahromi, M. Mosalanejad, and B. Deghhan, "Design and modeling remote monitoring system for a solar power plant," in Proc. 2009 2nd Int. Conf. Comput. Elect. Eng., IEEE Comput. Soc., pp. 81-86, DOI: 10.1109/ICCEE.2009.163.

[15] M. A. Azim, S. M. Ariful Huda, H. Mohammad, and N. Amin, "Microcontroller based standalone PV system for wireless sensor node," in Proc. Int. Conf. Comput. Commun. Eng., Kuala Lumpur,Malayasia, May 13-15, 2008, pp. 1136-1139.

[16] M. Magno, D. Brunelli, L. Thiele, and L. Benini, "Adaptive power control for solar harvesting multimodal wireless smart camera," in Proc. 3rd ACM/IEEE Int. Conf. Distrib. Smart Cameras, ICDSC, 2009, pp. 1-7, DOI: 10.1109/ICDSC.2009.5289358.

[17] S. Karunanithi, N. M. Din, H. Hakinie, C. K. Hua, R. C. Omar, and T. C. Yee, "Performance of labscale solar powered wireless landfill monitoring system," in Proc. 3rd Int. Conf. Energy and Environ., ICEE, Malacca, Malaysia, Dec. 7-8, 2009, pp. 443-448.

[18] S. Masuda and T. Hattpri, "Development of a wireless remote monitoring system utilizing multiple wireless sensors," Electron. Commun. Japan, vol. 91, no. 6, pp. 61-0-617, 2008, (Translated from Denki Gakkai Ronbunshi, vol. 127-D, No. 6, 2007, pp. 610617). 
[19] J. G. Ju, I. G. Park, Y. W. Lee, J. S. Cho, H. W. Cho, H. Yoe, and C. S. Shin, “An implementation of the salt-farm monitoring system using wireless sensor network," in Security-Enriched Urban Computing and Smart Grid Communications in Computer and Information Science. Berlin, Germany: Springer-Verlag, 2010, vol. 78, pp. 647-655, DOI: 10.1007/978-3-642-16444-6_81.

[20] C. Alippi, R. Camplani, C. Galperti, and M. Reveri, "A robust, adaptive, solar-powered WSN framework for aquatic environmental monitoring," IEEE Sensors J., vol. 11, no. 1, pp. 45-55, Jan. 2011.

[21] C. Alippi and C. Galperti, "An adaptive system for optimal solar energy harvesting in wireless sensor network nodes," IEEE-Trans. Circuits Syst. I: Regular Papers, vol. 55, no. 6, pp. 1742-1750, Jul. 2008. 2590 IEEE SENSORS JOURNAL, VOL. 11, NO. 10, OCTOBER 2011

Chagitha Ranhotigamage received the Bachelor of Engineering degree from Massey University, Palmerston North, New Zealand. Currently, he is working towards the M.S. degree at Massey University. He has specialized in electronics and computer systems engineering. He has been an exchange student at the University of Rostock, Germany, under the New Zealand European Union Joint Mobility project for five months.

Subhas Chandra Mukhopadhyay (SM'02-F'11) graduated from the Department of Electrical Engineering, Jadavpur University, Calcutta, India, in 1987, with a Gold medal and received the Master of Electrical Engineering degree from Indian Institute of Science, Bangalore, India, in 1989 and the Ph.D. (Eng.) degree from Jadavpur University, India, in 1994, and the Doctor of Engineering degree from Kanazawa University, Kanazawa, Japan, in 2000. Currently, he is working as an Associate Professor with the School of Engineering and Advanced Technology, Massey University, Palmerston North, New Zealand. He has over 21 years of teaching and research experiences. He has authored/coauthored over 240 papers in different international journals, conferences, and book chapters. He has edited nine conference proceedings. He has also edited eight special issues of international journals as lead guest editor and ten books out of which eight are with SpringerVerlag. His fields of interest include sensors and sensing technology, electromagnetics, control, electrical machines, and numerical field calculation, etc.

Dr. Mukhopadhyay was awarded numerous awards throughout his career and attracted over NZ \$3.5 M on different research projects. He is a Fellow of IEE (U.K.), an Associate Editor of the IEEE SENSORS JOURNAL and the IEEE TRANSACTIONS ON INSTRUMENTATION AND MEASUREMENTS. He is on the Editorial Board of the e-Journal on Non-Destructive Testing, Sensors and Transducers, the Transactions on Systems, Signals and Devices (TSSD), the Journal on the Patents on Electrical Engineering, and the Journal of Sensors. He is the Coeditor-in-Chief of the International Journal on Smart Sensing and Intelligent Systems (www.s2is.org). He is on the Technical Program Committee of the IEEE Sensors Conference, the IEEE IMTC Conference, the IEEE DELTA Conference and numerous other conferences. He was the Technical Program Chair of ICARA 2004, ICARA 2006, and ICARA 2009. He was the General Chair/Co-Chair of ICST 2005, ICST 2007, IEEE ROSE 2007, IEEE EPSA 2008, ICST 2008, IEEE Sensors 2008, ICST 2010, and IEEE Sensors 2010. He has organized the IEEE Sensors Conference 2009 at Christchurch, New Zealand, during October 25-28, 2009, as General Chair. He is the Chair of the IEEE Instrumentation and Measurement Society, New Zealand Chapter. He is a Distinguished Lecturer of the IEEE Sen 